hep-ph/0506176

KEK-TH-1023

June 2005

\title{
Single and Pair Production of Doubly Charged Higgs Bosons at Hadron Colliders
}

\author{
A.G. Akeroyd ${ }^{*}$ and Mayumi Aoki ${ }^{\dagger}$ \\ KEK Theory Group, \\ 1-1 Oho \\ Tsukuba, Japan 305-0801
}

\begin{abstract}
Current searches for doubly charged Higgs bosons $\left(H^{ \pm \pm}\right)$at the Fermilab Tevatron are sensitive to single production of $H^{ \pm \pm}$, although the pair production mechanism $q \bar{q} \rightarrow$ $H^{++} H^{--}$is assumed to be dominant. In the context of a Higgs Triplet Model we study the mechanism $q^{\prime} \bar{q} \rightarrow H^{ \pm \pm} H^{\mp}$ at the Tevatron and CERN Large Hadron Collider, and show that its inclusion can significantly improve the search potential for $H^{ \pm \pm}$. Moreover, assuming that the neutrino mass is generated solely by the triplet field Yukawa coupling to leptons, we compare the branching ratios of $H^{ \pm \pm} \rightarrow l^{ \pm} l^{ \pm}$and $H^{ \pm \pm} \rightarrow H^{ \pm} W^{*}$ for the cases of a normal hierarchical, inverted hierarchical and degenerate neutrino mass spectrum.
\end{abstract}

PACS index :12.60.Fr,14.80.Cp,14.60.Pq

Keywords : Higgs boson, Neutrino mass and mixing

*akeroyd@post.kek.jp

†mayumi.aoki@kek.jp 


\section{Introduction}

The quest for Higgs bosons is of utmost importance at high energy colliders [1, [2], 3]. In the Standard Model (SM), one isospin $I=1 / 2$, hypercharge $Y=1$ complex scalar doublet breaks the Electroweak Symmetry (EW) and provides mass for the fermions, $W^{ \pm}$and $Z$. One neutral scalar, $\phi^{0}$, remains as a physical degree of freedom - "the SM Higgs boson". Such a framework predicts $\rho\left(=M_{W}^{2} / M_{Z}^{2} \cos ^{2} \theta_{W}\right)=1$ at tree-level, a result which is in impressive agreement with the experimental measurement of $\rho \approx 1$ [4]. More generally, any Higgs sector composed solely of $I=1 / 2, Y=1$ doublets assures $\rho=1$ at tree-level, with calculable 1-loop corrections [5].

Predicting $\rho=1$ at tree-level is certainly an attractive feature of $I=1 / 2, Y=1$ doublet representations, although models with isospin triplets $(I=1)$ can also be considered [1]. Such models have various virtues and deficiencies. If the neutral member of the triplet acquires a vacuum expectation value $(\mathrm{VEV})$ then $\rho=1$ at tree-level is no longer guaranteed, and the triplet VEV must be very small in order to comply with the measured value $\rho \approx 1$. However, unlike doublets, $Y=2$ triplets can give rise to neutrino masses and mixings whose magnitude is proportional to the triplet vacuum expectation value multiplied by an arbitrary Yukawa coupling $\left(h_{i j}\right)$ without invoking a right handed neutrino [6], 7].

A clear phenomenological signature of $Y=2$ triplets would be the observation of a doubly charged Higgs boson $H^{ \pm \pm}$. Such $H^{ \pm \pm}$have been searched for at the $e^{+} e^{-}$collider LEP, resulting in mass limits of the order $m_{H^{ \pm \pm}}>100 \mathrm{GeV}$ [8], [9], [10, [11]. Their existence can also affect a wide variety of processes, such as Bhabha scattering, the anomalous magnetic moment of the muon $(g-2)_{\mu}$, and lepton flavour violating $\mu^{ \pm}$and $\tau^{ \pm}$decays [12, [13, [14, [15], 16, [17]. The Fermilab Tevatron recently performed the first search for $H^{ \pm \pm}$at hadron colliders. The production process $p \bar{p} \rightarrow \gamma, Z \rightarrow H^{++} H^{--}$was assumed, with subsequent decay $H^{ \pm \pm} \rightarrow$ $l^{ \pm} l^{ \pm}$. D0 [18] searched for $H^{ \pm \pm} \rightarrow \mu^{ \pm} \mu^{ \pm}$while CDF [19] searched for 3 final states $H^{ \pm \pm} \rightarrow$ $\mu^{ \pm} \mu^{ \pm}, \mu^{ \pm} e^{ \pm}$and $e^{ \pm} e^{ \pm}$. Mass limits of the order $m_{H^{ \pm \pm}}>130 \mathrm{GeV}$ were obtained with an integrated luminosity of $240 \mathrm{pb}^{-1}$, assuming $\mathrm{BR}\left(H^{ \pm \pm} \rightarrow l_{i}^{ \pm} l_{j}^{ \pm}\right)=100 \%$ [19] in a given channel. These are the strongest direct mass limits on any type of Higgs boson, which shows the strong search capability of hadron colliders in the channel $H^{ \pm \pm} \rightarrow l^{ \pm} l^{ \pm}$.

Given this strong search potential, in this paper we consider the phenomenological effect of relaxing these simplifying assumptions for the dominant production mechanism and decay modes of $H^{ \pm \pm}$. Although work along these lines has appeared previously [14, [20, 21], 22], [23, 24] we develop and expand the preceding analyses. For example, if $h_{i j}$ are solely responsible for the currently favoured form of the neutrino mass matrix then $\operatorname{BR}\left(H^{ \pm \pm} \rightarrow l_{i}^{ \pm} l_{j}^{ \pm}\right)<100 \%$ in a given channel [14. In this paper we study in detail the alternative production mechanism $q^{\prime} \bar{q} \rightarrow W^{*} \rightarrow H^{ \pm \pm} H^{\mp}$ [24], which can be as large as $q \bar{q} \rightarrow \gamma, Z \rightarrow H^{++} H^{--}$. Since the current search strategy at the Tevatron is in fact sensitive to single production of $H^{ \pm \pm}$, we introduce the inclusive single production cross-section $\left(\sigma_{H^{ \pm \pm}}\right)$as the sum of the single and pair production cross-sections. We point out that the contribution of $q^{\prime} \bar{q} \rightarrow W^{*} \rightarrow H^{ \pm \pm} H^{\mp}$ to $\sigma_{H^{ \pm \pm}}$strengthens the Tevatron mass limit on $H^{ \pm \pm}$, which in general has a dependence on $m_{H^{ \pm}}$. Moreover, we quantify the impact of the potentially important decay mode $H^{ \pm \pm} \rightarrow H^{ \pm} W^{*}[22$ in the light

of recent neutrino data. Although such a decay can weaken the $H^{ \pm \pm}$search capability in the leptonic channel, observation of $H^{ \pm \pm} \rightarrow H^{ \pm} W^{*}$ together with one or more leptonic channels might permit an order of magnitude estimate of $h_{i j}$ [21], [23]. 
Our work is organized as follows. In Section 2 we introduce the Higgs Triplet Model. In Section 3 we study the production mechanism $q^{\prime} \bar{q} \rightarrow H^{ \pm \pm} H^{\mp}$ and its phenomenological effect on the $H^{ \pm \pm}$search at the Tevatron and LHC. In Section 4 we quantify the impact of the decay $H^{ \pm \pm} \rightarrow H^{ \pm} W^{*}$, while Section 5 considers the search potential of the Tevatron in the generalized scenario. Finally, in Section 6 we present our conclusions.

\section{The Higgs Triplet Model}

Higgs $I=1$ triplet representations arise in several well motivated models of physics beyond the SM [1],20]. For example, Left-Right (L-R) symmetric models built on the gauge group $S U(2)_{R} \times S U(2)_{L} \times U(1)$ contain both left- and right-handed $I=1, Y=2$ triplet representations. Such models also require extra gauge bosons and can provide naturally light neutrino masses via the seesaw mechanism. Little Higgs models [25] also require $I=1, Y=2$ triplet representations, as well as new gauge bosons and fermions. However, Higgs triplets can be considered as a minimal addition to the SM [26]- for a review see [27]. We will focus on a particularly simple model [6], 7] which merely adds a $I=1, Y=2$ complex (left-handed) Higgs triplet to the SM Lagrangian, hereafter referred to as the "Higgs Triplet Model" or "HTM". Such a model can provide a Majorana mass for the observed neutrinos without the need for a right handed neutrino via the gauge invariant Yukawa interaction: ${ }^{1}$

$$
\mathcal{L}=h_{i j} \psi_{i L}^{T} C i \tau_{2} \Delta \psi_{j L}+h . c
$$

Here $h_{i j}(i, j=1,2,3)$ is an arbitrary coupling, $C$ is the Dirac charge conjugation operator, $\psi_{i L}=\left(\nu_{i}, l_{i}\right)_{L}^{T}$ is a left-handed lepton doublet, and $\Delta$ is a $2 \times 2$ representation of the $Y=2$ complex (left-handed) triplet fields:

$$
\Delta=\left(\begin{array}{cc}
\Delta^{+} / \sqrt{2} & \Delta^{++} \\
\Delta^{0} & -\Delta^{+} / \sqrt{2}
\end{array}\right)
$$

The Higgs potential [14] is as follows, with $\Phi=\left(\phi^{+}, \phi^{0}\right)^{T}$ :

$$
\begin{aligned}
V= & m^{2}\left(\Phi^{\dagger} \Phi\right)+\lambda_{1}\left(\Phi^{\dagger} \Phi\right)^{2}+M^{2} \operatorname{Tr}\left(\Delta^{\dagger} \Delta\right)+\lambda_{2}\left[\operatorname{Tr}\left(\Delta^{\dagger} \Delta\right)\right]^{2}+\lambda_{3} \operatorname{Det}\left(\Delta^{\dagger} \Delta\right) \\
& +\lambda_{4}\left(\Phi^{\dagger} \Phi\right) \operatorname{Tr}\left(\Delta^{\dagger} \Delta\right)+\lambda_{5}\left(\Phi^{\dagger} \tau_{i} \Phi\right) \operatorname{Tr}\left(\Delta^{\dagger} \tau_{i} \Delta\right)+\left(\frac{1}{\sqrt{2}} \mu\left(\Phi^{T} i \tau_{2} \Delta^{\dagger} \Phi\right)+\text { h.c }\right)
\end{aligned}
$$

The term $\mu \Phi \Delta \Phi$, where $\mu$ is a dimensionful trilinear coupling, gives rise to a VEV $v_{\Delta}$ for the neutral member of the triplet $\Delta^{0}$ :

$$
v_{\Delta} \simeq \mu v^{2} / 2 M^{2}
$$

Here $M$ is the common triplet mass $\left(M^{2} \Delta^{\dagger} \Delta\right)$. Since we are interested in the case of light triplets we take $M \approx v$, and so $v_{\Delta} \approx \mu$. A non-zero $v_{\Delta}$ gives rise to the following mass matrix for neutrinos:

$$
m_{i j}=2 h_{i j}\left\langle\Delta^{0}\right\rangle=\sqrt{2} h_{i j} v_{\Delta}
$$

\footnotetext{
${ }^{1}$ Note that the analogous term for a $Y=0$ triplet is forbidden by gauge invariance
} 
Note that the HTM is free from a massless Goldstone boson (Majoron) arising from the violation of the lepton number $(L)$ global symmetry, because the Higgs potential contains the term $\mu \Phi \Delta \Phi$ term which explicitly violates lepton number when $\Delta$ is assigned $L=-2$. Cosmological data provides a constraint on the neutrino masses $m_{i}, \Sigma m_{i} \lesssim 0.75 \mathrm{eV}$ [28]. Lepton flavour violating (LFV) processes involving $\mu$ and $\tau$ provide the strongest upper limits on $h_{i j}$ and hence $v_{\Delta}$ cannot be arbitrarily small if the HTM is to accommodate the currently favoured form of the neutrino mass matrix. A rough lower bound $v_{\Delta} \gtrsim 10 \mathrm{eV}$ can be derived. An upper limit on $v_{\Delta}$ can be obtained from considering its effect on $\rho$. In the HTM $\rho$ is given by (where $x=v_{\Delta} / v$ ):

$$
\rho \equiv 1+\delta \rho=\frac{1+2 x^{2}}{1+4 x^{2}}
$$

From the measurement of $\rho \approx 1$ a purely tree-level analysis gives the bound $v_{\Delta} / v \lesssim 0.03$. We will comment on the 1-loop expression for $\delta \rho$ below [29, 30], 31]. In this paper we will assume

$$
10 \mathrm{eV} \lesssim v_{\Delta} \lesssim 10000 \mathrm{eV}
$$

Hence the tree-level value of $\rho$ is essentially equal to 1 , thus easily satisfying the experimental constraint on $\delta \rho$. Such small values of $v_{\Delta}$ can be explained by a 2 loop mechanism [14 or in the context of extra dimensions [32, [33]. Moreover, such values of $v_{\Delta}$ would permit some $h_{i j}$ to be sufficiently large to enhance various LFV $\mu$ and $\tau$ decays to the sensitivity of current and forthcoming experiments [14, [15], [17], and are also consistent with the requirement that any primordially generated baryon asymmetry is not erased by the lepton number violating triplet interactions [34].

The HTM has 7 Higgs bosons $\left(H^{++}, H^{--}, H^{+}, H^{-}, H^{0}, A^{0}, h^{0}\right)$. While $H^{ \pm \pm}$is purely triplet $\left(=\Delta^{ \pm \pm}\right)$, the remaining eigenstates would in general be mixtures of the doublet and triplet fields. Such mixing is proportional to the triplet VEV, and hence small even if $v_{\Delta}$ assumes its largest value of a few $\mathrm{GeV}$. Therefore the first six eigenstates are essentially composed of triplet fields, while the $I=1 / 2$ doublet gives rise to a SM like $h^{0}$ and the Goldstone bosons $G^{ \pm}, G^{0}$. The most striking signature of the HTM would be the observation of $H^{ \pm \pm}{ }^{2}$ In the HTM there exists the following relationships among the masses of the physical Higgs bosons:

$$
\begin{array}{r}
m_{H^{ \pm \pm}}^{2} \simeq M^{2}+2 \frac{\left(\lambda_{4}-\lambda_{5}\right)}{g^{2}} M_{W}^{2} \\
m_{H^{ \pm}}^{2} \simeq m_{H^{ \pm \pm}}^{2}+2 \frac{\lambda_{5}}{g^{2}} M_{W}^{2} \\
m_{H^{0}, A^{0}}^{2} \simeq m_{H^{ \pm}}^{2}+2 \frac{\lambda_{5}}{g^{2}} M_{W}^{2}
\end{array}
$$

Here $M$ is the triplet mass term, while $\lambda_{4}, \lambda_{5}$ are dimensionless quartic couplings. For $\lambda_{5}>0$ $\left(\lambda_{5}<0\right)$ one has the following hierarchy $m_{H^{ \pm \pm}}<m_{H^{ \pm}}<m_{H^{0}, A^{0}}\left(m_{H^{ \pm \pm}}>m_{H^{ \pm}}>m_{H^{0}, A^{0}}\right)$. Clearly $M$ sets the scale for the mass of the triplet fields, while the mass splitting among the

\footnotetext{
${ }^{2}$ The dominantly triplet eigenstates $H^{ \pm}, H^{0}$ and $A^{0}$ can have a different phenomenology to the analogous Higgs bosons in doublet $(I=1 / 2, Y=1)$ representations.
} 
eigenstates is determined by the quartic couplings and can be $\mathcal{O}\left(M_{W}\right)$. We will focus on Higgs boson masses of interest for the Tevatron and LHC, and hence we assume $M \gtrsim 1 \mathrm{TeV}$.

At the 1-loop level the Higgs sector contribution to $\delta \rho$ is a function of $v_{\Delta}$ and the Higgs boson masses. Although a quantitative analysis in the context of the HTM is still lacking, explicit formulae for the contributions of $Y=2$ triplets to the self-energies of the $W$ and $Z$ in the context of L-R symmetric models and Little Higgs Models can be found in [29], 30]. In particular, such contributions are sensitive to the mass splittings of the Higgs bosons. In the HTM the triplet Higgs boson mass splitting is determined by the quartic coupling $\lambda_{5}$, with $\lambda_{5}=0$ giving rise to degenerate triplet scalars of mass $M$. We will present results for both the degenerate case and for mild splittings of up to $20 \mathrm{GeV}$ in our discussion of $H^{ \pm \pm}$phenomenology at the Tevatron.

We now briefly discuss present mass bounds on the Higgs bosons of the HTM, which differ in some cases from the commonly quoted mass bounds in the 2HDM. If $H^{0}$ and $A^{0}$ were the lightest, they could have been produced at LEP via the mechanism $e^{+} e^{-} \rightarrow A^{0} H^{0}$ (note that $e^{+} e^{-} \rightarrow Z H^{0}$ is proportional to $v_{\Delta}$ and hence negligible). However, since $A^{0}$ and $H^{0}$ would both decay invisibly to $\nu \bar{\nu}$, Ref. 35] suggested using LEP data on $\gamma \nu \bar{\nu}$ (where $\gamma$ arises from bremsstrahlung from $e^{+}$or $\left.e^{-}\right)$and derived the mass limit $m_{H^{0}, A^{0}} \gtrsim 55 \mathrm{GeV}$. Concerning $H^{ \pm}$, LEP searched for $H^{ \pm} \rightarrow c s$ or $\tau \nu_{\tau}$ which are expected to be the dominant decays in doublet models, and obtained mass limits around $m_{H^{ \pm}} \gtrsim 80 \mathrm{GeV}$. For the triplet $H^{ \pm}$the decays $H^{ \pm} \rightarrow e^{ \pm} \nu, \mu^{ \pm} \nu$ may have large BRs. However, in this scenario one could presumably use data from slepton searches $e^{+} e^{-} \rightarrow \tilde{l}^{+} \tilde{l}^{-} \rightarrow l^{+} l^{-} \chi^{0} \bar{\chi}^{0}$ to derive similar mass limits ( $\gtrsim 80$ $\mathrm{GeV}$ ) [36]. A recent quantitative analysis of the above decays in the context of a Little Higgs Model can be found in [37.

Concerning $H^{ \pm \pm}$, LEP searched for both left-handed $H_{L}^{ \pm \pm}$and right-handed $H_{R}^{ \pm \pm}$(which we will not consider in this paper) via several mechanisms:

(i) Pair production via $e^{+} e^{-} \rightarrow \gamma^{*}, Z^{*} \rightarrow H^{++} H^{--}$followed by decay to $l^{+} l^{+} l^{-} l^{-}\left(l^{ \pm}=\right.$ $\left.e^{ \pm}, \mu^{ \pm}, \tau^{ \pm}\right)$; the cross-section is determined by gauge couplings and leads to mass limits of $m_{H^{ \pm \pm}}>100 \mathrm{GeV}$ [8], [9], 10].

(ii) Single production of $H^{ \pm \pm}$via $e^{+} e^{-} \rightarrow H^{ \pm \pm} e^{\mp} e^{\mp}$; the rate is determined by the coupling $h_{11}$ and leads to excluded regions in the plane $\left(h_{11}, m_{H^{ \pm \pm}}\right)$, with sensitivity up to $m_{H^{ \pm \pm}} \lesssim 180 \mathrm{GeV}$. Limits of $10^{-2} \rightarrow 10^{-1}$ were set on $h_{11}$ [1].

(iii) The effect of $H^{ \pm \pm}$on Bhabha scattering $e^{+} e^{-} \rightarrow e^{+} e^{-}$; as in (ii) above this leads to excluded regions in the plane $\left(h_{11}, m_{H^{ \pm \pm}}\right)$[10, 11] with sensitivity up to $m_{H^{ \pm \pm}} \lesssim 2 \mathrm{TeV}$. Limits of $10^{-2} \rightarrow 10^{-1}$ were set on $h_{11}$.

The direct searches for $H^{ \pm \pm}$will continue at the hadron colliders, Tevatron and LHC.

\section{Production of $H^{ \pm \pm}$at the Tevatron}

A distinct signature of $H^{ \pm \pm}$would be a pair of same sign charged leptons $\left(e^{ \pm}\right.$or $\left.\mu^{ \pm}\right)$with high invariant mass. At hadron colliders such a signal has a relatively high detection efficiency and 
enjoys essentially negligible background from Standard Model processes. Earlier theoretical studies of the search potential for $H^{ \pm \pm}$at such colliders can be found in [20, [21], with a recent analysis at the LHC in 38. The decays of $H^{ \pm \pm}$to states involving $\tau^{ \pm}$are more problematic at hadron colliders, although simulations in these channels [21, 38. promise sensitivity to values of $m_{H^{ \pm \pm}}$beyond the LEP limits. The decays $H^{ \pm \pm} \rightarrow W^{ \pm} W^{ \pm}$are proportional to $v_{\Delta}$, and can be neglected in the case of very small $v_{\Delta}$ of interest to us.

In 2003 the Tevatron performed the first search for $H^{ \pm \pm}$at a hadron collider. D0 [18, have searched for $H^{ \pm \pm} \rightarrow \mu^{+} \mu^{-}$while CDF [19] searched for 3 final states: $H^{ \pm \pm} \rightarrow e^{ \pm} e^{ \pm}, e^{ \pm} \mu^{ \pm}, \mu^{ \pm} \mu^{ \pm}$. The assumed production mechanism for $H^{ \pm \pm}$is $q \bar{q} \rightarrow \gamma^{*}, Z^{*} \rightarrow H^{++} H^{--} .{ }^{3}$ This cross-section depends on only one unknown parameter, $m_{H^{ \pm \pm}}$, and importantly is not suppressed by any small factor such as a Yukawa coupling $h_{i j}$ or a triplet VEV. The search assumes that $H^{ \pm \pm}$is sufficiently long-lived to decay in the detector, which corresponds to $h_{l l}>10^{-5}$. A search for a long lived $H^{ \pm \pm}$decaying outside the detector has been performed in [39]. The cross-section also depends on the hypercharge of the Higgs representation, which is $Y=2$ in the HTM. This value of $Y$ is also assumed in the experimental searches. The explicit partonic cross-section at leading order $(\mathrm{LO})$ is as follows (where $q=u, d)$ :

$$
\sigma_{L O}\left(q \bar{q} \rightarrow H^{++} H^{--}\right)=\frac{\pi \alpha^{2}}{9 Q^{2}} \beta_{1}^{3}\left[e_{q}^{2} e_{H}^{2}+\frac{e_{q} e_{H} \mathrm{v}_{q} \mathrm{v}_{H}\left(1-M_{Z}^{2} / Q^{2}\right)+\left(\mathrm{v}_{q}^{2}+\mathrm{a}_{q}^{2}\right) \mathrm{v}_{H}^{2}}{\left(1-M_{Z}^{2} / Q^{2}\right)^{2}+M_{Z}^{2} \Gamma_{Z}^{2} / Q^{4}}\right]
$$

Here $\mathrm{v}_{q}=\left(I_{3 q}-2 e_{q} s_{W}^{2}\right) /\left(s_{W} c_{W}\right), \mathrm{a}_{q}=I_{3 q} /\left(s_{W} c_{W}\right)$, and $\mathrm{v}_{H}=\left(I_{3 H}-e_{H} s_{W}^{2}\right) /\left(s_{W} c_{W}\right)$. The third isospin component is denoted by $I_{3 q}\left(I_{3 H}\right)$ and $e_{q}\left(e_{H}\right)$ is the electric charge of the quark $q\left(H^{ \pm \pm}\right) . s_{W}$ and $c_{W}$ are $\sin \theta_{W}$ and $\cos \theta_{W}$ respectively. $Q^{2}$ is the partonic centre-of-mass (COM) energy. $\alpha$ is the QED coupling evaluated at the scale $Q, M_{Z}$ is the $Z$ boson mass, $\Gamma_{Z}$ is the $Z$ boson width, and $\beta_{1}=\sqrt{1-4 m_{H^{ \pm \pm}}^{2} / Q^{2}}$. Order $\alpha_{s}$ QCD corrections modify the LO cross-section by a factor $K \approx 1.3$ at the Tevatron for $m_{H^{ \pm \pm}}<200 \mathrm{GeV}$, and $K \approx 1.25$ at the LHC for $m_{H^{ \pm \pm}}<1000 \mathrm{GeV}$ [40]. We neglect the gluon-gluon fusion $\left(\alpha_{s}^{2}\right)$ contribution to $H^{++} H^{--}$production, which has no compensatory enhancement factor analogous to the $\tan ^{4} \beta$ term for doublet $H^{ \pm}$production via $g g \rightarrow H^{+} H^{-}$[41].

Assuming that $H^{ \pm \pm}$production proceeds via this pair production process, the absence of signal enables a limit to be set on the product:

$$
\sigma\left(p \bar{p} \rightarrow H^{++} H^{--}\right) \times B R\left(H^{ \pm \pm} \rightarrow l_{i}^{ \pm} l_{j}^{ \pm}\right)
$$

Clearly the strongest constraints on $m_{H^{ \pm \pm}}$are obtained assuming $\operatorname{BR}\left(H^{ \pm \pm} \rightarrow l_{i}^{ \pm} l_{j}^{ \pm}\right)=$ $100 \%$. Currently these mass limits stand at: $133,115,136 \mathrm{GeV}$ for the $e^{ \pm} e^{ \pm}, e^{ \pm} \mu^{ \pm}, \mu^{ \pm} \mu^{ \pm}$channels respectively [19]. In the HTM one expects $B R\left(H^{ \pm \pm} \rightarrow l_{i}^{ \pm} l_{j}^{ \pm}\right) \neq 100 \%$ if Eqn.(5) is required to explain the currently favoured form of the neutrino mass matrix [14.

The current search strategy is in fact sensitive to any singly produced $H^{ \pm \pm}$, i.e. signal candidates are events with one pair of same sign leptons reconstructing to $m_{H^{ \pm \pm}}$. This requirement is sufficient to reduce the SM background to negligible proportions. Hence the search potential of the Tevatron merely depends on the signal efficiencies for the signal (currently

${ }^{3}$ The model-dependent contribution from any $Z^{\prime}$ (which can enhance the cross-section [24, [38) is currently not considered. 
$\approx 34 \%, 34 \%, 18 \%$ for $\mu \mu, e e, e \mu)$ and the integrated luminosity. With these relatively high efficiencies and an expected $\mathcal{L}=4-8 f b^{-1}$ by the year 2009 , discovery with $>5$ events will be possible for $\sigma_{H^{++} H^{--}}$of a few fb, which corresponds to a mass reach $m_{H^{ \pm \pm}}<200 \mathrm{GeV}$.

Although single $H^{ \pm \pm}$production processes such as $p \bar{p} \rightarrow W^{ \pm} \rightarrow W^{\mp} H^{ \pm \pm}$can be neglected ${ }^{4}$ due to the strong triplet VEV suppression, the mechanism $p \bar{p} \rightarrow W^{*} \rightarrow H^{ \pm \pm} H^{\mp}$ is potentially sizeable. This latter process proceeds via a gauge coupling constant and is not suppressed by any small factor. The LO partonic cross-section is as follows:

$$
\sigma_{L O}\left(q^{\prime} \bar{q} \rightarrow H^{++} H^{-}\right)=\frac{\pi \alpha^{2}}{144 s_{W}^{4} Q^{2}} C_{T}^{2} p_{W}^{2} \beta_{2}^{3}
$$

Here $C_{T}$ arises from the $H^{ \pm \pm} H^{\mp} W^{\mp}$ vertex and $C_{T}=2$ for $I=1, Y=2$ triplet fields (the doublet component of $H^{ \pm}$is negligible); $\beta_{2}=\sqrt{\left(1-\left(m_{H^{ \pm}}+m_{H^{ \pm \pm}}\right)^{2} / Q^{2}\right)\left(1-\left(m_{H^{ \pm}}-m_{H^{ \pm \pm}}\right)^{2} / Q^{2}\right)}$ and $p_{W}=Q^{2} /\left(Q^{2}-M_{W}^{2}\right)$. For simplicity, we take the same $K=1.3$ as for $\sigma\left(q \bar{q} \rightarrow H^{++} H^{--}\right)$ at the Tevatron and $K=1.25$ at the LHC. Explicit calculations for the $K$ factor for the process $\sigma\left(q^{\prime} \bar{q} \rightarrow H^{ \pm} A^{0}\right)$ in the MSSM [44] (which shares the same $K$ factor as $q^{\prime} \bar{q} \rightarrow H^{++} H^{-}$) give $K \approx 1.2$. In this paper we will study in detail the magnitude and relative importance of $\sigma\left(q^{\prime} \bar{q} \rightarrow H^{ \pm \pm} H^{\mp}\right)$. Although we work in the HTM, our numerical analysis is relevant for other models which possess a $I=1, Y=2$ Higgs triplet (e.g. L-R symmetric models and Little Higgs Models).

A previous quantitative study of this mechanism can be found in 24]. Cross-sections were given at both LHC and Tevatron energies for $m_{H^{ \pm \pm}}>200 \mathrm{GeV}$ with the simplifying assumption $m_{H^{ \pm \pm}}=m_{H^{ \pm}}$. It was shown that $\sigma\left(q^{\prime} \bar{q} \rightarrow H^{ \pm \pm} H^{\mp}\right)$ can of comparable size to $\sigma(q \bar{q} \rightarrow$ $\left.H^{++} H^{--}\right)$.

In this paper we first generalize the work of [24] as follows:

(i) In our discussion at the Tevatron we consider masses in the range $100 \mathrm{GeV}<m_{H^{ \pm \pm}}<200$ $\mathrm{GeV}$ which will be probed during Run II, and allow mild mass splittings $\left|m_{H^{ \pm \pm}}-m_{H^{ \pm}}\right| \leq$ $20 \mathrm{GeV}$.

(ii) In our discussion at the LHC we consider larger mass splittings $\left|m_{H^{ \pm \pm}}-m_{H^{ \pm}}\right| \leq 80 \mathrm{GeV}$.

(iii) For both the Tevatron and LHC we study in detail the relative magnitude of $\sigma\left(q^{\prime} \bar{q} \rightarrow\right.$ $\left.H^{ \pm \pm} H^{\mp}\right)$ and $\sigma\left(q \bar{q} \rightarrow H^{++} H^{--}\right)$.

Moreover, motivated by the fact that the currently employed Tevatron search strategy is sensitive to single production of $H^{ \pm \pm}$, we advocate the use of the inclusive single production cross-section $\left(\sigma_{H^{ \pm \pm}}\right)$when comparing the experimentally excluded region with the theoretical cross-section. This leads to a strengthening of the mass bound for $m_{H^{ \pm \pm}}$which now carries a dependence on $m_{H^{ \pm}}$. We introduce the single production cross-section as follows:

$$
\sigma_{H^{ \pm \pm}}=\sigma\left(p \bar{p}, p p \rightarrow H^{++} H^{--}\right)+\sigma\left(p \bar{p}, p p \rightarrow H^{++} H^{-}\right)+\sigma\left(p \bar{p}, p p \rightarrow H^{--} H^{+}\right)
$$

\footnotetext{
${ }^{4}$ Single production of a right-handed triplet via $q^{\prime} \bar{q} \rightarrow W_{R}^{ \pm} \rightarrow W_{R}^{\mp} H^{ \pm \pm}$[42] and $W_{R}^{ \pm} W_{R}^{ \pm}$fusion [4] can be sizeable at the LHC.
} 
(a)

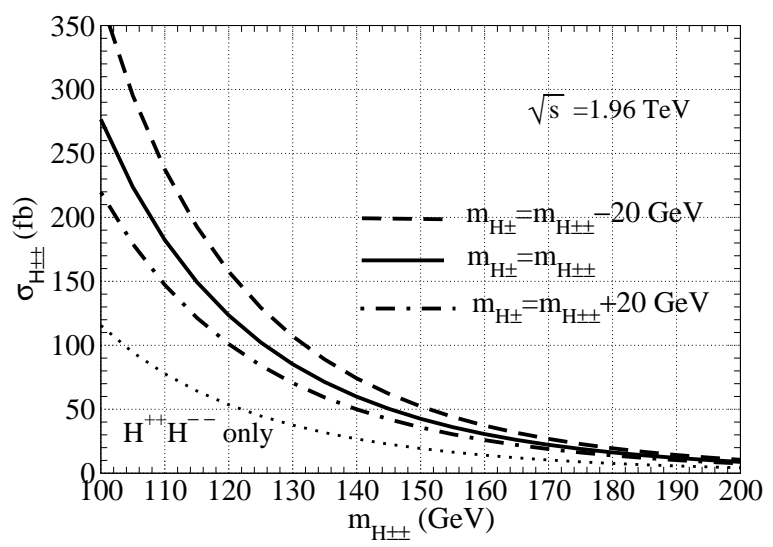

(b)

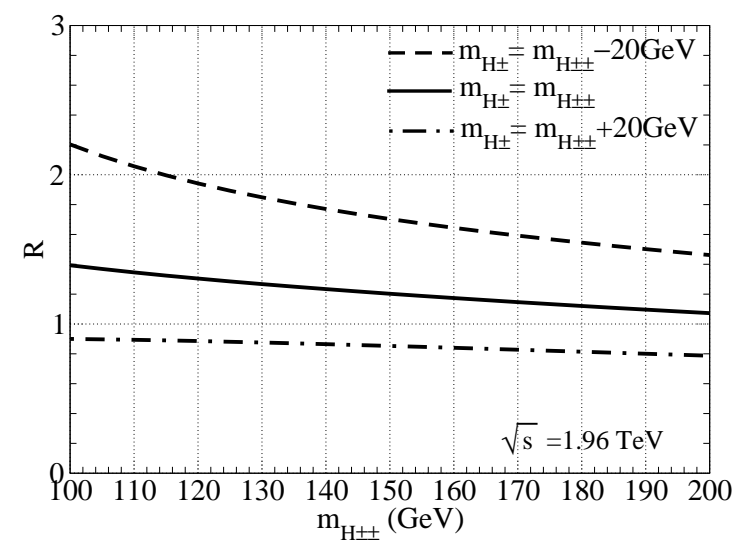

Figure 1: (a) Single production cross-section of $H^{ \pm \pm}\left(\sigma_{H^{ \pm \pm}}\right)$at the Tevatron as a function of $m_{H^{ \pm \pm}}$for different values of $m_{H^{ \pm}}$. (b) Ratio $R$ as a function of $m_{H^{ \pm \pm}}$. We use CTEQ6L1 parton distribution functions.

At the Tevatron $\sigma\left(p \bar{p} \rightarrow H^{++} H^{-}\right)=\sigma\left(p \bar{p} \rightarrow H^{--} H^{+}\right)$while at the LHC $\sigma\left(p p \rightarrow H^{++} H^{-}\right)>$ $\sigma\left(p p \rightarrow H^{--} H^{+}\right)$. If a signal for $H^{ \pm \pm}$were found in the 2 lepton channel, subsequent searches could select signal events with 3 or 4 leptons, in order to disentangle $q \bar{q} \rightarrow H^{++} H^{--}$and $q^{\prime} \bar{q} \rightarrow H^{ \pm \pm} H^{\mp}$. In our numerical analysis we utilize the CTEQ6L1 parton distribution functions (pdfs) [45. We take the factorization scale $(Q)$ as the partonic COM energy $(\sqrt{s})$. Our results for $\sigma\left(q \bar{q} \rightarrow H^{++} H^{--}\right)$agree with those in [21, 40]. Our results for $\sigma\left(q^{\prime} \bar{q} \rightarrow H^{ \pm \pm} H^{\mp}\right)$ agree with those in [24] (and taking $C_{T}=1$ agree with $\sigma\left(q^{\prime} \bar{q} \rightarrow H^{ \pm} A^{0}\right)$ in the 2HDM/MSSM [44]). The above cross-sections evaluated with MRST02 pdfs [46] agree with those evaluated with CTEQ6L1 to within $10 \% \rightarrow 15 \%$.

In Fig. 1 (a) we plot $\sigma_{H^{ \pm \pm}}$as a function of $m_{H^{ \pm \pm}}$at the Tevatron for three different values of $m_{H^{ \pm}}$. We take $K=1.3$. The current excluded regions from the $e^{ \pm} e^{ \pm}, e^{ \pm} \mu^{ \pm}, \mu^{ \pm} \mu^{ \pm}$searches correspond to the area above horizontal lines at roughly 40,70,35 fb respectively. The present mass limits for $m_{H^{ \pm \pm}}$are where the curve for $\mathrm{H}^{++} \mathrm{H}^{--}$intersects with the above horizontal lines, and read as $133,115,136 \mathrm{GeV}$ respectively for $\mathrm{BR}\left(H^{ \pm \pm} \rightarrow l_{i}^{ \pm} l_{j}^{ \pm}\right)=100 \%$. With the inclusion of the $H^{ \pm \pm} H^{\mp}$ channel, these mass limits increase to $150,130,150$ for $m_{H^{ \pm}}=m_{H^{ \pm \pm}}+20$ $\mathrm{GeV}$, strengthening to $160,140,160$ for $m_{H^{ \pm}}=m_{H^{ \pm \pm}}-20 \mathrm{GeV}$. Clearly the search potential of the Tevatron (i.e. the mass limit on $m_{H^{ \pm \pm}}$) increases significantly when one includes the contribution to $\sigma_{H^{ \pm \pm}}$from $p \bar{p} \rightarrow H^{ \pm \pm} H^{\mp}$. Note that the above mass limits strictly apply to the case when $H^{ \pm \pm}$decays leptonically, and with $\mathrm{BR}=100 \%$ in a given channel. However, if $h_{i j}$ are to provide the currently favoured form of the neutrino mass matrix then $\operatorname{BR}\left(H^{ \pm \pm} \rightarrow l_{i}^{ \pm} l_{j}^{ \pm}\right)<$ $100 \%$ in a given channel. Moreover, if $m_{H^{ \pm \pm}}>m_{H^{ \pm}}$then the decay channel $H^{ \pm \pm} \rightarrow H^{ \pm} W^{*}$ would be open. As shown in [22], this decay can be sizeable and thus reduces $\operatorname{BR}\left(H^{ \pm \pm} \rightarrow l_{i}^{ \pm} l_{j}^{ \pm}\right)$. We will return to these issues in Section 5.

In Fig. 1 (b) we plot the ratio of cross-sections $R$ at the Tevatron as a function of $m_{H^{ \pm \pm}}$, 
where $R$ is defined as follows:

$$
R \equiv \frac{\sigma\left(p \bar{p}, p p \rightarrow H^{++} H^{-}\right)+\sigma\left(p \bar{p}, p p \rightarrow H^{--} H^{+}\right)}{\sigma\left(p \bar{p}, p p \rightarrow H^{++} H^{--}\right)}
$$

The $m_{H^{ \pm \pm}}$dependence arises from the phase space functions $\beta_{1}$ and $\beta_{2}$ in Eqns 9 and [1], As can be seen, $0.8<R<2.2$ and thus $q^{\prime} \bar{q} \rightarrow H^{ \pm \pm} H^{\mp}$ contributes significantly to $\sigma_{H^{ \pm \pm}}$.

In Fig. 2 we plot the analogies of Fig.1 for the LHC. In Fig. 2 (a) we plot $\sigma_{H^{ \pm \pm}}$for 3 values of $m_{H^{ \pm \pm}}$and for larger mass splittings $\left(\left|m_{H^{ \pm \pm}}-m_{H^{ \pm}}\right| \leq 80 \mathrm{GeV}\right)$ than in Fig. 2. We take $K=1.25$. As before, the inclusion of $q^{\prime} \bar{q} \rightarrow H^{ \pm \pm} H^{\mp}$ significantly increases the search potential e.g. if sensitivity to $\sigma_{H^{ \pm \pm}}=1 \mathrm{fb}$ is attained, the mass reach extends from $m_{H^{ \pm \pm}}<600 \mathrm{GeV}$ $\left(H^{++} H^{--}\right.$only) to $750 \mathrm{GeV}$ for $\left(m_{H^{ \pm}}=m_{H^{ \pm \pm}}-80 \mathrm{GeV}\right)$. Recently 38] performed a simulation of the detection prospects at the LHC for $q \bar{q} \rightarrow H^{++} H^{--}$for the cases where 3 and 4 leptons are detected. With $100 \mathrm{fb}^{-1}$, sensitivity to $m_{H^{ \pm \pm}} \lesssim 800 \mathrm{GeV}$ (3 leptons) and $m_{H^{ \pm \pm}} \lesssim 700 \mathrm{GeV}$ (4 leptons) is expected. We are not aware of a simulation for the case where only 2 leptons are detected. Presumably even larger values of $m_{H^{ \pm \pm}}(\gtrsim 800 \mathrm{GeV})$ could be probed. In Fig. 2 (b) we plot $R$ as a function of $m_{H^{ \pm \pm}}$. One can see that $R>1$ for the upper two curves for all $m_{H^{ \pm \pm}}$, while for the lower curve $R>1$ for $m_{H^{ \pm \pm}}>260 \mathrm{GeV}$. Note that the dependence of $R$ on $m_{H^{ \pm \pm}}$differs from that observed in Fig. 1 (b), which can be attributed to the different parton luminosity functions at the Tevatron and LHC.

(a)

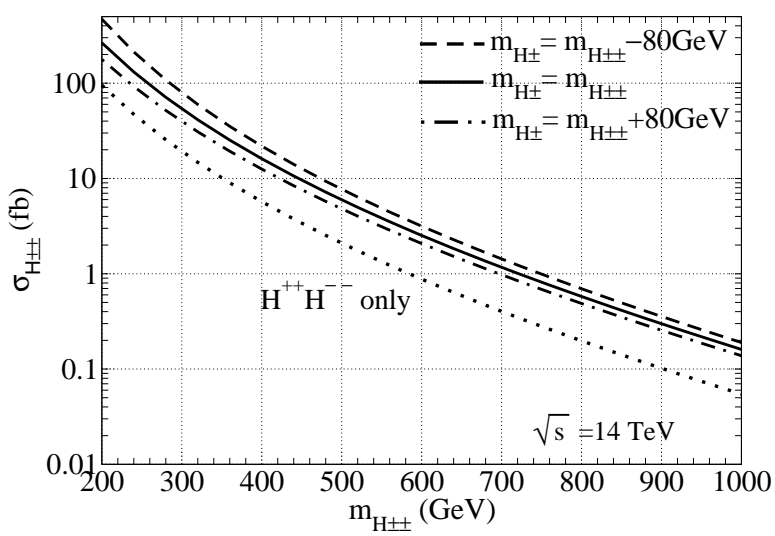

(b)

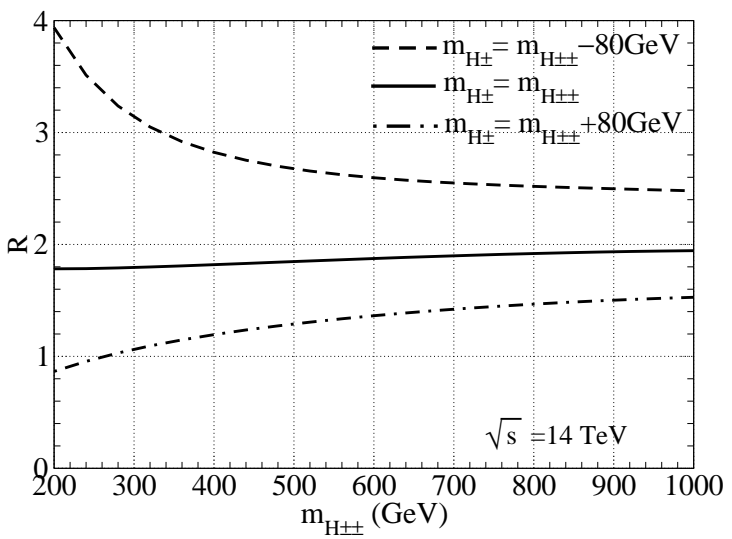

Figure 2: (a) Single production cross-section of $H^{ \pm \pm}\left(\sigma_{H^{ \pm \pm}}\right)$at the LHC as a function of $m_{H^{ \pm \pm}}$ for different values of $m_{H^{ \pm}}$. (b) Ratio $R$ as a function of $m_{H^{ \pm \pm}}$. We use CTEQ6L1 parton distribution functions. 


\section{Neutrino mass hierarchy and the decay $H^{ \pm \pm} \rightarrow H^{ \pm} W^{*}$}

The current experimental searches assume that the sole decay mode of $H^{ \pm \pm}$is $H^{ \pm \pm} \rightarrow l_{i}^{ \pm} l_{j}^{ \pm}$ mediated by the arbitrary Yukawa couplings $h_{i j}$. The decay rate for $H^{ \pm \pm} \rightarrow l_{i}^{ \pm} l_{j}^{ \pm}$is given by:

$$
\Gamma\left(H^{ \pm \pm} \rightarrow l_{i}^{ \pm} l_{j}^{ \pm}\right)=S \frac{m_{H^{ \pm \pm}}}{8 \pi}\left|h_{i j}\right|^{2}
$$

where $S=1(2)$ for $i=j(i \neq j)$. Clearly $\Gamma\left(H^{ \pm \pm} \rightarrow l_{i}^{ \pm} l_{j}^{ \pm}\right)$depends crucially on the absolute value of the $h_{i j}$, although the leptonic BRs are determined by the relative values. In this section we consider the impact of the decay mode $H^{ \pm \pm} \rightarrow H^{ \pm} W^{*}$ on the BRs of the leptonic channels. It has been known for some time that $\operatorname{BR}\left(H^{ \pm \pm} \rightarrow H^{ \pm} W^{*}\right)$ is potentially sizeable and a quantitative analysis can be found in [22]. The decay rate for $H^{ \pm \pm} \rightarrow H^{ \pm} W^{*}$ (summing over all fermion states for $W^{*} \rightarrow f f$ excluding the $t$ quark) is given by:

$$
\Gamma\left(H^{ \pm \pm} \rightarrow H^{ \pm} W^{*}\right)=9 G_{F}^{2} M_{W}^{4} m_{H^{ \pm \pm}} C_{T}^{2} P /\left(16 \pi^{3}\right)
$$

where $P$ is the phase space term (which we calculate by numerical integration) and $C_{T}(=2$ ) is from the coupling $H^{ \pm \pm} H^{ \pm} W . P$ depends on the mass difference $\Delta m$ defined by $\Delta m=$ $m_{H^{ \pm \pm}}-m_{H^{ \pm}}$, and $P=0$ for $\Delta m=0$. If $m_{H^{ \pm}}<m_{H^{ \pm \pm}}$this decay can compete with $H^{ \pm \pm} \rightarrow l_{i}^{ \pm} l_{j}^{ \pm}$since the phase space suppression of the virtual $W^{*}$ is compensated by the gauge strength coupling [21]. Ref. 22] showed that $H^{ \pm \pm} \rightarrow H^{ \pm} W^{*}$ can dominate over $H^{ \pm \pm} \rightarrow l_{i}^{ \pm} l_{j}^{ \pm}$ if $\Delta m$ is sizeable $(>40 \mathrm{GeV})$ and $h_{i j}$ are of order $10^{-3}$ or less. A large $\operatorname{BR}\left(H^{ \pm \pm} \rightarrow H^{ \pm} W^{*}\right)$ would debilitate the $H^{ \pm \pm}$search potential in the leptonic channel. However, as emphasized in [23], observation of $H^{ \pm \pm} \rightarrow H^{ \pm} W^{*}$ together with one or more of the leptonic channels could provide information on the absolute values of $h_{i j}$. If only $\operatorname{BR}\left(H^{ \pm \pm} \rightarrow l_{i}^{ \pm} l_{j}^{ \pm}\right)$are measured then only the relative values of the $h_{i j}$ can be evaluated. The decay rate for $H^{ \pm \pm} \rightarrow H^{ \pm} W^{*}$ is theoretically calculable once $m_{H^{ \pm}}$and $m_{H^{ \pm \pm}}$are known experimentally, and thus it can be used as a benchmark decay with which to estimate the total width of $H^{ \pm \pm}$. It is known that the BRs of the leptonic channels depend on which solution to the neutrino mass matrix is realized [14. However, a quantitative analysis of the impact of $H^{ \pm \pm} \rightarrow H^{ \pm} W^{*}$ in the various allowed scenarios is still lacking and will be presented below. We are not aware of any experimental simulation of $H^{ \pm \pm} \rightarrow H^{ \pm} W^{*}$. The signature would depend crucially on the decay products of $H^{ \pm}$, which are are either $H^{ \pm} \rightarrow l^{ \pm} \nu_{l}$ (driven by $h_{i j}$ ), or possibly $H^{ \pm} \rightarrow H^{0} W^{*}, A^{0} W^{*}$.

We now briefly review relevant results and formulae from neutrino physics. The neutrino mass matrix is diagonalized by the MNS (Maki-Nakagawa-Sakata) matrix $V_{\text {MNS }}$ [47. Using Eq.(15) one can write the couplings $h_{i j}$ as follows:

$$
h_{i j}=\frac{1}{\sqrt{2} v_{\Delta}} V_{\mathrm{MNS}} \operatorname{diag}\left(m_{1}, m_{2}, m_{3}\right) V_{\mathrm{MNS}}^{T}
$$

Here we take the basis in which the unitary matrix responsible for diagonalizing the chargedlepton mass matrix is a unit matrix. The MNS matrix in the standard parametrization is as follows:

$$
V_{\mathrm{MNS}}=\left(\begin{array}{ccc}
c_{1} c_{3} & s_{1} c_{3} & s_{3} e^{-i \delta} \\
-s_{1} c_{2}-c_{1} s_{2} s_{3} e^{i \delta} & c_{1} c_{2}-s_{1} s_{2} s_{3} e^{i \delta} & s_{2} c_{3} \\
s_{1} s_{2}-c_{1} c_{2} s_{3} e^{i \delta} & -c_{1} s_{2}-s_{1} c_{2} s_{3} e^{i \delta} & c_{2} c_{3}
\end{array}\right)\left(\begin{array}{ccc}
1 & 0 & 0 \\
0 & e^{i \varphi_{1} / 2} & 0 \\
0 & 0 & e^{i \varphi_{2} / 2}
\end{array}\right)
$$


where $s_{i} \equiv \sin \theta_{i}$ and $c_{i} \equiv \cos \theta_{i}, \delta$ is the Dirac phase and $\varphi_{1}$ and $\varphi_{2}$ are the Majorana phases.

Neutrino oscillation experiments involving solar [4], atmospheric [4] and reactor neutrinos [50] are sensitive to the mass-squared differences and the mixing angles. and give the following preferred values:

$$
\begin{gathered}
\Delta m_{12}^{2} \equiv m_{2}^{2}-m_{1}^{2} \simeq 8.0 \times 10^{-5} \mathrm{eV}^{2}, \quad\left|\Delta m_{13}^{2}\right| \equiv\left|m_{3}^{2}-m_{1}^{2}\right| \simeq 2.1 \times 10^{-3} \mathrm{eV}^{2} \\
\sin ^{2} 2 \theta_{1} \simeq 0.8, \quad \sin ^{2} 2 \theta_{2} \simeq 1, \quad \sin ^{2} 2 \theta_{3} \lesssim 0.16 .
\end{gathered}
$$

Since the sign of $\Delta m_{13}^{2}$ and the mass of the lightest neutrino are both undetermined at present, distinct neutrino mass hierarchy patterns are classified as follows: Normal hierarchy $(\mathrm{NH})$ $\left(m_{1}<m_{2} \ll m_{3}\right)$, Inverted hierarchy $(\mathrm{IH})\left(m_{2}>m_{1} \gg m_{3}\right)$, Quasi-degenerate (DG) $\left(m_{1} \sim\right.$ $m_{2} \sim m_{3} \gg \sqrt{\left|\Delta m_{13}^{2}\right|}$. From Eq.(15) and Eq.(16) it can be shown that:

$$
\sum_{i, j} h_{i j}^{2} v_{\Delta}^{2} \propto \sum_{i} m_{i}^{2}
$$

Hence the total leptonic decay width depends on the absolute mass of the neutrinos, and the value of $\sum_{i} m_{i}^{2}$ depends on which solution to the neutrino mass matrix (NH,IH,DG) is realized. The minimum value of $\sum_{i} m_{i}^{2}$ is $\left|\Delta m_{13}^{2}\right|$ while the maximum is given by the cosmological constraint.

In Fig 3 we show contours of $\operatorname{BR}\left(H^{ \pm \pm} \rightarrow H^{ \pm} W^{*}\right)$ in the plane $\left(m_{H^{ \pm \pm}}, v_{\Delta}\right)$, for three different solutions to the neutrino mass matrix. We assume that $m_{1(3)}=0$ for $\mathrm{NH}(\mathrm{IH})$ and $m_{1}=0.2$ $\mathrm{eV}$ for DG. We take $m_{H^{ \pm}}=m_{H^{ \pm \pm}}-20 \mathrm{GeV}$. From Eq 16, all $h_{i j}$ are determined once $v_{\Delta}$ is specified. In order to comply with current experimental upper limits on LFV decays of $\mu^{ \pm}$ and $\tau^{ \pm}$, one can derive the bound $v_{\Delta}>10 \mathrm{eV}$ for $\mathrm{NH}$ and $\mathrm{IH}$, and $v_{\Delta}>100 \mathrm{eV}$ for DG. The stronger constraint on $v_{\Delta}$ in DG arises because $\sum_{i} m_{i}$ in DG is larger than those in NH and IH.
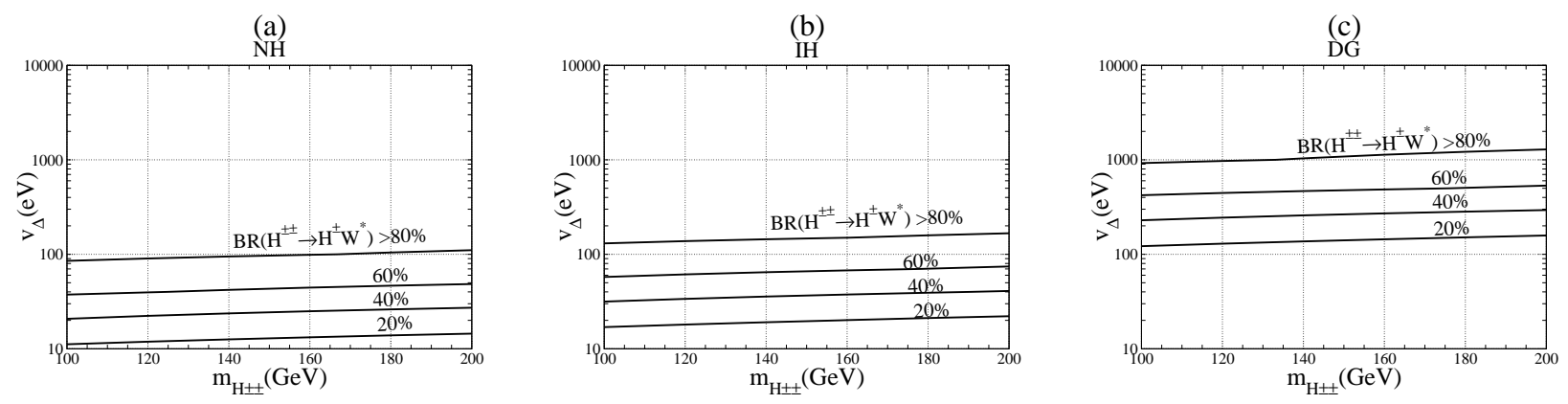

Figure 3: Contours of $\operatorname{BR}\left(H^{ \pm \pm} \rightarrow H^{ \pm} W^{*}\right)$ in the plane $\left(m_{H^{ \pm \pm}}, v_{\Delta}\right)$, for NH (a), IH (b) and DG (c). We take $m_{H^{ \pm}}=m_{H^{ \pm \pm}}-20 \mathrm{GeV}$.

From Fig 3 it is clear that $\operatorname{BR}\left(H^{ \pm \pm} \rightarrow H^{ \pm} W^{*}\right)$ can be sizeable, and approaches $100 \%$ for larger $v_{\Delta}$. For a fixed value of $v_{\Delta}$, one can see that $\mathrm{BR}\left(H^{ \pm \pm} \rightarrow H^{ \pm} W^{*}\right)$ is relatively more important in $\mathrm{NH}$ and $\mathrm{IH}$ than in DG. This can be understood from Eq. 20, since DG requires heavier neutrinos (and thus larger $h_{i j}$ ) which in turn reduces $\operatorname{BR}\left(H^{ \pm \pm} \rightarrow H^{ \pm} W^{*}\right)$. One can consider three distinct scenarios with very different magnitudes for $\operatorname{BR}\left(H^{ \pm \pm} \rightarrow H^{ \pm} W^{*}\right)$ and $\mathrm{BR}\left(H^{ \pm \pm} \rightarrow l^{ \pm} l^{ \pm}\right)$ 
(i) $\operatorname{BR}\left(H^{ \pm \pm} \rightarrow H^{ \pm} W^{*}\right) \gg \mathrm{BR}\left(H^{ \pm \pm} \rightarrow l^{ \pm} l^{ \pm}\right)$:

In this case the current search strategy (which requires $H^{ \pm \pm} \rightarrow l^{ \pm} l^{ \pm}$decay) is ineffective. Simulations have not been carried out for the decay $H^{ \pm \pm} \rightarrow H^{ \pm} W^{*}$ although one might naively expect sensitivity comparable to that for the decay $H^{ \pm \pm} \rightarrow \tau^{ \pm} \tau^{ \pm}$, as suggested in [21], 23].

(ii) $\operatorname{BR}\left(H^{ \pm \pm} \rightarrow H^{ \pm} W^{*}\right) \approx \operatorname{BR}\left(H^{ \pm \pm} \rightarrow l^{ \pm} l^{ \pm}\right)$:

The search for $H^{ \pm \pm} \rightarrow l^{ \pm} l^{ \pm}$would be effective and $H^{ \pm \pm}$could be discovered in one or more leptonic channels. If $H^{ \pm \pm} \rightarrow H^{ \pm} W^{*}$ is also observed then information on the absolute value of $h_{i j}$ might be possible: Using Eqs 14 and 15, the ratio of leptonic events $\left(N_{l_{i} l_{j}}\right)$ to $H^{ \pm} W^{*}$ events $\left(N_{H^{ \pm} W^{*}}\right)$ is given as follows:

$$
\frac{N_{l_{i} l_{j}}}{N_{H^{ \pm} W^{*}}} \sim \frac{h_{i j}^{2}}{P}
$$

Observation of the leptonic channel provides $m_{H^{ \pm \pm}}$. If $m_{H^{ \pm}}$can be roughly measured then $P$ (and hence the partial width for $H^{ \pm \pm} \rightarrow H^{ \pm} W^{*}$ ) can be calculated. From the above equation one can obtain an order of magnitude estimate of $h_{i j}$.

(iii) $\operatorname{BR}\left(H^{ \pm \pm} \rightarrow H^{ \pm} W^{*}\right) \ll \operatorname{BR}\left(H^{ \pm \pm} \rightarrow l^{ \pm} l^{ \pm}\right)$:

In this case the current search strategy $\left(H^{ \pm \pm} \rightarrow l^{ \pm} l^{ \pm}\right)$is effective. If $\operatorname{BR}\left(H^{ \pm \pm} \rightarrow l_{i}^{ \pm} l_{j}^{ \pm}\right)$ are measured then the ratios of $h_{i j}$ can be evaluated. This can be compared with Eqn 5 in order to see which neutrino solution is realized [14. The absolute values of $h_{i j}$ cannot be measured unless a LFV decay of $\mu$ and/or $\tau$ is observed.

\section{Tevatron search potential in HTM}

We now study the search potential of the Tevatron for the generalized case in the HTM where $p \bar{p} \rightarrow H^{ \pm \pm} H^{\mp}$ is included, $\operatorname{BR}\left(H^{ \pm \pm} \rightarrow H^{ \pm} W^{*}\right) \neq 0 \%$ and $h_{i j}$ are required to reproduce a phenomenologically acceptable neutrino mass matrix. We relax the assumptions for the Majorana phases and take $\varphi_{1}, \varphi_{2}=0$ or $\pi$, which leads the 7 distinct solutions:

$$
\begin{aligned}
& \mathrm{NH}: \quad m_{1}<m_{2} \ll m_{3}, \\
& \text { IH1: } \quad m_{2}>m_{1} \gg m_{3}, \quad \text { IH2: } \quad-m_{2}>m_{1} \gg m_{3} \text {, } \\
& \text { DG1: } \quad m_{1} \simeq m_{2} \simeq m_{3}, \quad \text { DG2: } \quad m_{1} \simeq m_{2} \simeq-m_{3} \text {, } \\
& \text { DG3: } \quad m_{1} \simeq-m_{2} \simeq m_{3} \text {, } \quad \text { DG4: } m_{1} \simeq-m_{2} \simeq-m_{3} \text {. }
\end{aligned}
$$

In the HTM, BR $\left(H^{ \pm \pm} \rightarrow l^{ \pm} l^{ \pm}\right)$are predicted and different in each of the 7 distinct solutions $(\mathrm{NH}, \mathrm{IH} 1, \mathrm{IH} 2, \mathrm{DG} 1 \rightarrow \mathrm{DG} 4)$, and their ratios were evaluated in 14. Note that such predictions of $\operatorname{BR}\left(H^{ \pm \pm} \rightarrow l^{ \pm} l^{ \pm}\right)$are a feature of the HTM in which the couplings $h_{i j}$ are the sole origin of neutrino mass. This direct correlation between $\mathrm{BR}\left(H^{ \pm \pm} \rightarrow l^{ \pm} l^{ \pm}\right)$and the neutrino mass matrix may not extend to $H^{ \pm \pm}$of other models in which neutrinos can acquire mass by other means e.g. the seesaw mechanism in L-R models or by a combination of mechanisms which may or may not include the $h_{i j}$ couplings [37, [51. In contrast, the production process $\sigma\left(p \bar{p} \rightarrow H^{ \pm \pm} H^{\mp}\right)$ is certainly relevant in any model with $Y=2$ triplets. 
In Figs $4 \rightarrow$ we plot $\sigma_{l l}$ as a function of $m_{H^{ \pm \pm}}$, where $\sigma_{l l}$ is the total leptonic $(l=e, \mu, \tau)$ cross-section defined as:

$$
\sigma_{l l}=\sigma\left(p \bar{p} \rightarrow H^{++} H^{--}\right) \times B_{l l}\left(2-B_{l l}\right)+2 \sigma\left(p \bar{p} \rightarrow H^{++} H^{-}\right) \times B_{l l}
$$

The contribution to $\sigma_{l l}$ from $\sigma\left(p \bar{p} \rightarrow H^{++} H^{--}\right)$falls more slowly with decreasing $B_{l l}$ since signal candidates are events with at least 2 leptons. Eq. (22) simplifies to Eq 10 in the limit where $\sigma\left(p \bar{p} \rightarrow H^{ \pm \pm} H^{\mp}\right)=0$ and $B_{l l}=1$. Figs 《(a) shows $\sigma_{l l}$ for the NH with $m_{H^{ \pm}}=m_{H^{ \pm \pm}}$, which leads to $B_{l l}=1$. In this case $\sum \sigma_{l l}=\sigma_{H^{ \pm \pm}}$. For the other figures we take $m_{H^{ \pm}}=$ $m_{H^{ \pm \pm}}-20 \mathrm{GeV}$, which induces a sizeable (but not dominant) $\mathrm{BR}\left(H^{ \pm \pm} \rightarrow H^{ \pm} W^{*}\right)$, and hence $\sum \sigma_{l l}<\sigma_{H^{ \pm \pm}}$. We set $v_{\Delta}=10 \mathrm{eV}$ in Figs,4 and 5 and $v_{\Delta}=100 \mathrm{eV}$ in Figs 6. We only plot $\sigma_{l l}$ for $e e, e \mu, \mu \mu$ since the Tevatron has already performed searches in these channels. Sensitivity to $\sigma_{l l}$ of a few fb will be possible with the anticipated integrated luminosities of $4-8 \mathrm{fb}^{-1}$. There are plans to search for the 3 leptonic decays involving $\tau(e \tau, \mu \tau, \tau \tau)$ although the discovery reach in $m_{H^{ \pm \pm}}$is expected to be inferior to that for the ee, $e \mu, \mu \mu$ channels. In all figures we take $\theta_{3}=0^{\circ}$. From the figures it is clear that $\sigma_{e e, e \mu, \mu \mu}$ differ considerably in each of the 7 scenarios. Optimal coverage is for cases DG1 and DG4, which have $\sigma_{e e, \mu \mu} \geq 5 \mathrm{fb}$ and $\sigma_{e \mu, \mu \mu} \geq 5 \mathrm{fb}$ respectively for $m_{H^{ \pm \pm}} \lesssim 180 \mathrm{GeV}$. For $\mathrm{NH}, \sigma_{\mu \mu} \geq 5 \mathrm{fb}$ for $m_{H^{ \pm \pm}} \lesssim 190 \mathrm{GeV}$ but $\sigma_{e e}$ and $\sigma_{e \mu}$ are both unobservable. Taking $\theta_{3}$ at its largest experimentally allowed value results in minor changes to all figures, with the most noticeable effect being a significant reduction of $\sigma_{\mu \mu}$ in DG4. Clearly the Tevatron Run II not only has strong search potential for $H^{ \pm \pm}$, but is also capable of distinguishing between the various allowed scenarios for the neutrino mass matrix.

(a)

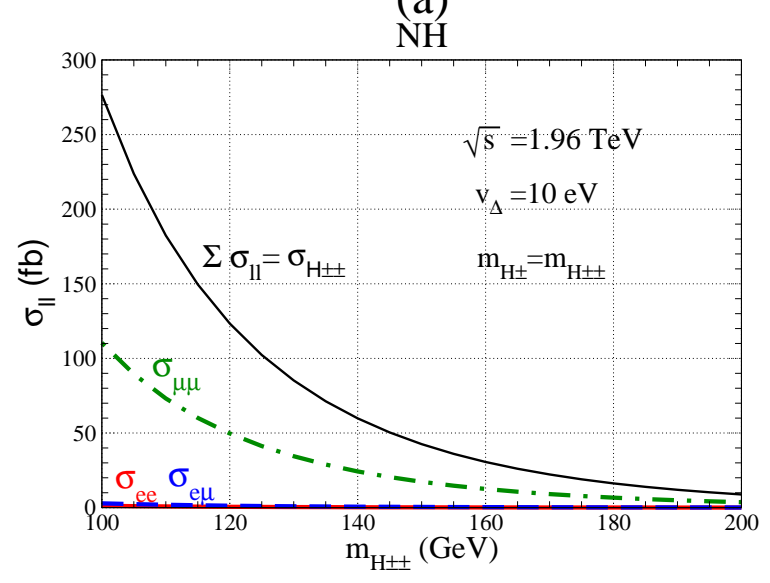

(b)

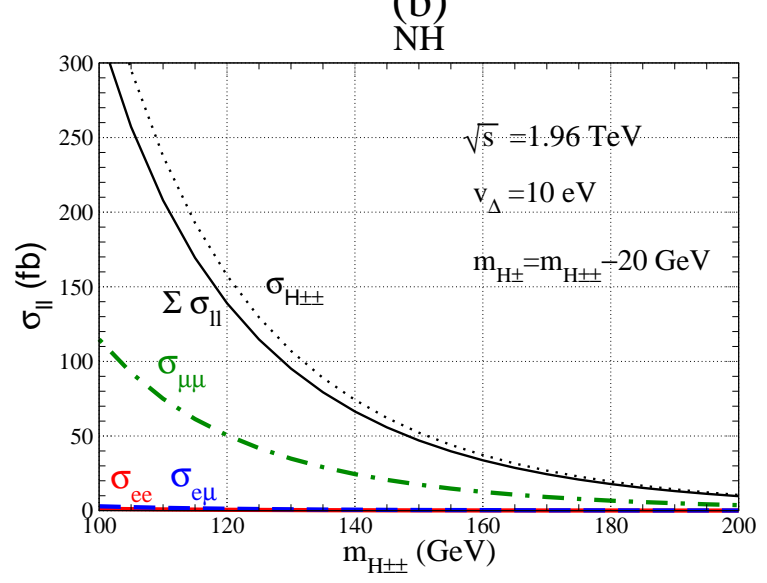

Figure 4: $\sigma_{l l}$ as a function of $m_{H^{ \pm \pm}}$for $\mathrm{NH}$ with (a) $m_{H^{ \pm}}=m_{H^{ \pm \pm}}$and (b) $m_{H^{ \pm}}=m_{H^{ \pm \pm}}-20$ $\mathrm{GeV}$. 
(a)

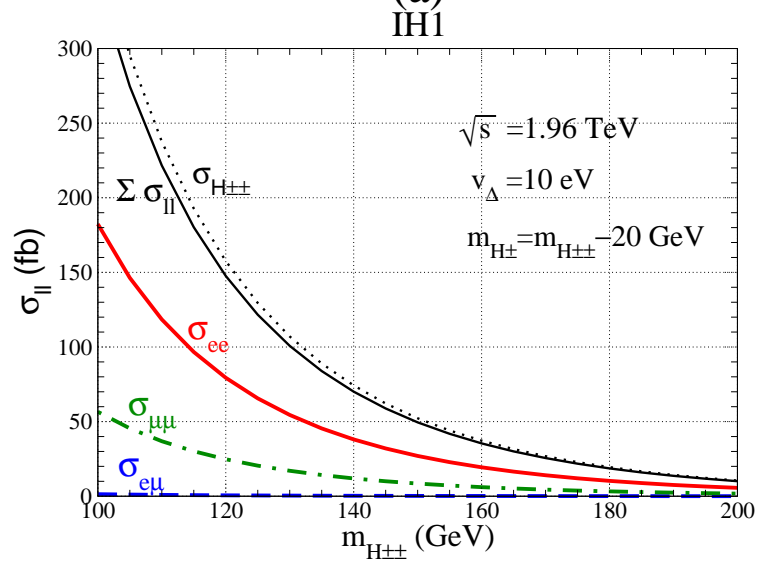

(b)

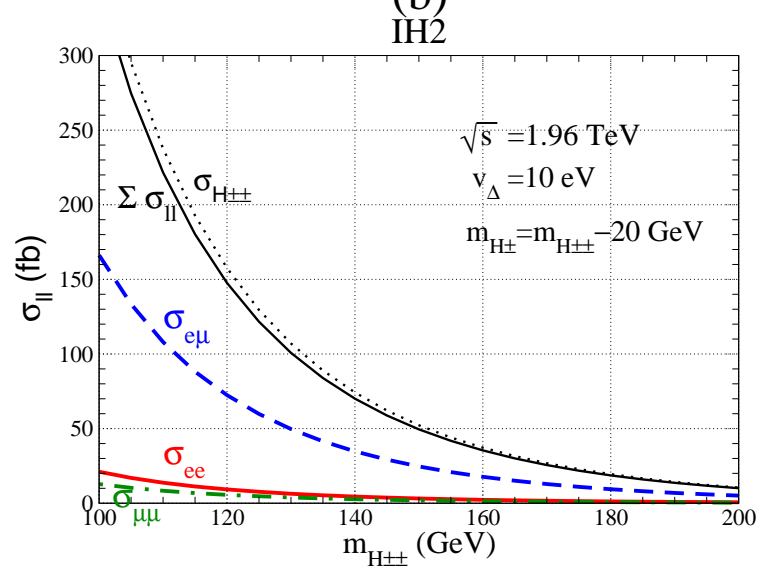

Figure 5: $\sigma_{l l}$ as a function of $m_{H^{ \pm \pm}}$for (a) IH1 and (b) IH2.

\section{Conclusions}

We have studied the production of doubly charged Higgs bosons $\left(H^{ \pm \pm}\right)$at hadron colliders in the Higgs Triplet Model (HTM), in which a complex $Y=2$ scalar triplet is added to the Standard Model. The HTM can explain the observed neutrino mass matrix by invoking Yukawa couplings $h_{i j}$ of the triplet fields to leptons. A definitive signal of the HTM would be the observation of the decay $H^{ \pm \pm} \rightarrow l^{ \pm} l^{ \pm}$, which enjoys almost negligible background at hadron colliders, and whose branching ratios are correlated with the neutrino mass matrix. We studied the production mechanism $q^{\prime} \bar{q} \rightarrow H^{ \pm \pm} H^{\mp}$ which can be as large as the mechanism $q \bar{q} \rightarrow H^{++} H^{--}$assumed in the current searches at the Tevatron. Since the present search strategy is sensitive to single production of $H^{ \pm \pm}$, we advocated the use of the inclusive single production cross-section $\left(\sigma_{H^{ \pm \pm}}\right)$when comparing the experimentally excluded region with the theoretical cross-section. This leads to a strengthening of the mass bound for $m_{H^{ \pm \pm}}$which now carries a dependence on $m_{H^{ \pm}}$, and significantly improves the $H^{ \pm \pm}$search potential at the Tevatron and LHC. Although we performed our numerical analysis in the HTM, we emphasized that the introduction of $\sigma_{H^{ \pm \pm}}$is also relevant for any model which contains a $Y=2$ Higgs triplet (e.g. L-R symmetric models and Little Higgs Models).

Moreover, we quantified the impact of the decay mode $H^{ \pm \pm} \rightarrow H^{ \pm} W^{*}$ for the case of a hierarchical, inverted hierarchical and degenerate neutrino mass spectrum. On discovering a $H^{ \pm \pm}$it would be imperative to measure the absolute value of $h_{i j}$ (and hence $v_{\Delta}$ ) in order to reconstruct the low energy Higgs triplet Lagrangian. We stressed that an order of magnitude estimate of $h_{i j}$ could be obtained if the channel $H^{ \pm \pm} \rightarrow H^{ \pm} W^{*}$ is observed and $m_{H^{ \pm}}$is roughly measured. We encourage a detailed experimental simulation of this decay mode at both the Tevatron and LHC. 
(a)

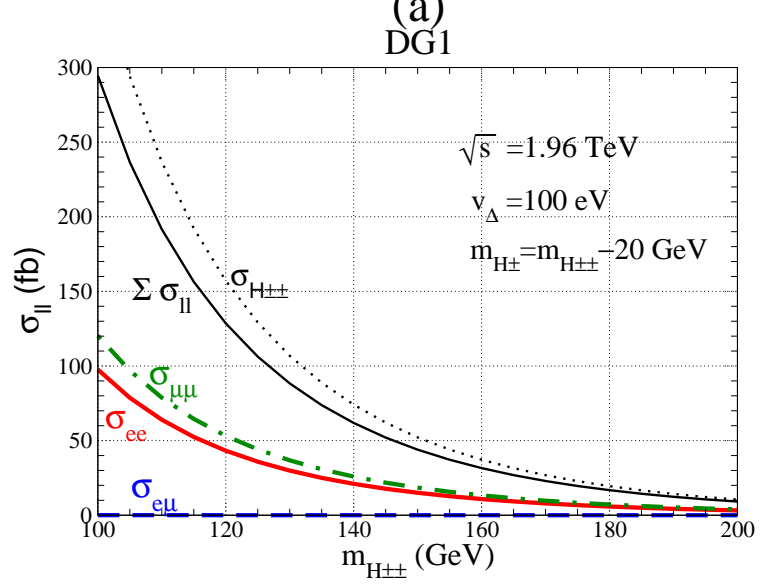

(c)

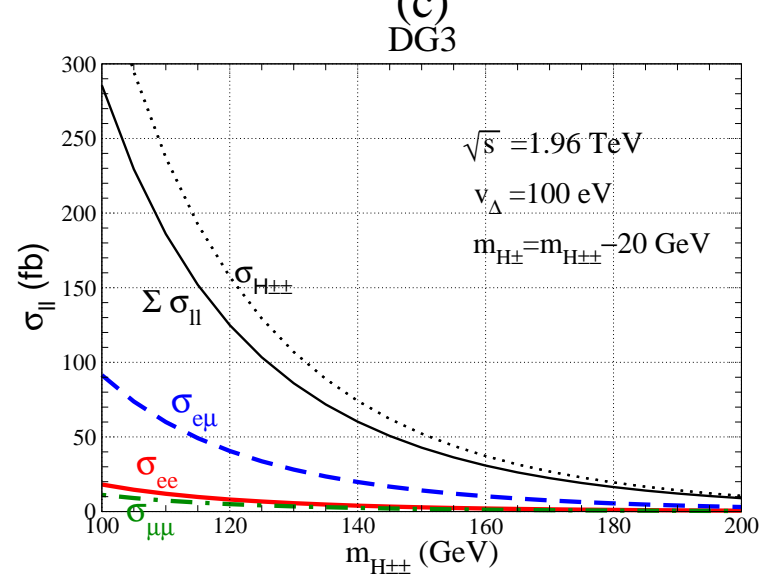

(b)

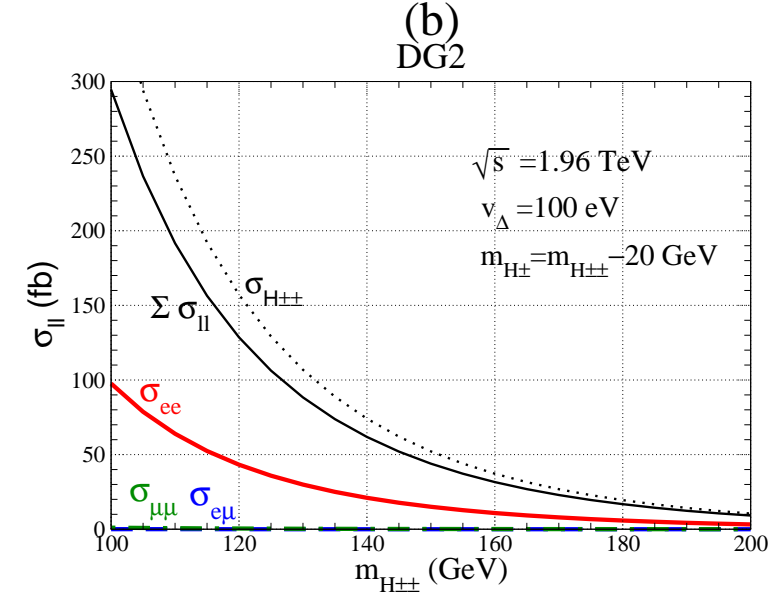

(d)

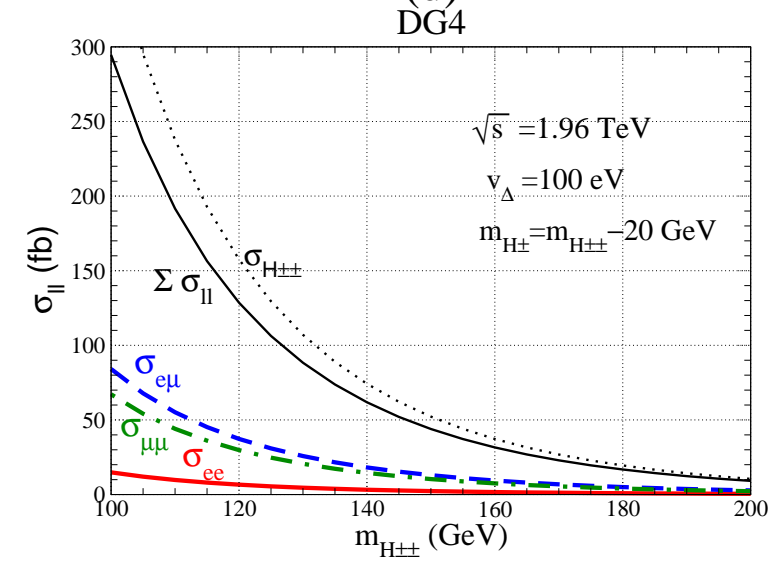

Figure 6: $\sigma_{l l}$ as a function of $m_{H^{ \pm \pm}}$for (a) DG1, (b) DG2, (c) DG3 and (d) DG4.

\section{References}

[1] J. F. Gunion, H. E. Haber, G. L. Kane and S. Dawson, "The Higgs Hunter's Guide," (Addison-Wesley Publishing Company, Redwood City, CA, 1990).

[2] M. Carena and H. E. Haber, Prog. Part. Nucl. Phys. 50, 63 (2003).

[3] A. Djouadi, arXiv:hep-ph/0503172; A. Djouadi, arXiv:hep-ph/0503173; V. Buscher and K. Jakobs, Int. J. Mod. Phys. A 20, 2523 (2005).

[4] S. Eidelman et al. [Particle Data Group], Phys. Lett. B 592, 1 (2004).

[5] W. Hollik, Z. Phys. C 32, 291 (1986); W. Hollik, Z. Phys. C 37, 569 (1988).

[6] J. Schechter and J. W. F. Valle, Phys. Rev. D 22, 2227 (1980).

[7] G. B. Gelmini and M. Roncadelli, Phys. Lett. B 99, 411 (1981). 
[8] J. Abdallah et al. [DELPHI Collaboration], Phys. Lett. B 552, 127 (2003).

[9] G. Abbiendi et al. [OPAL Collaboration], Phys. Lett. B 526, 221 (2002).

[10] P. Achard et al. [L3 Collaboration], Phys. Lett. B 576, 18 (2003).

[11] G. Abbiendi et al. [OPAL Collaboration], Phys. Lett. B 577, 93 (2003).

[12] M. L. Swartz, Phys. Rev. D 40, 1521 (1989).

[13] F. Cuypers and S. Davidson, Eur. Phys. J. C 2, 503 (1998).

[14] E. J. Chun, K. Y. Lee and S. C. Park, Phys. Lett. B 566, 142 (2003).

[15] M. Kakizaki, Y. Ogura and F. Shima, Phys. Lett. B 566, 210 (2003).

[16] S. Atag and K. O. Ozansoy, Phys. Rev. D 68, 093008 (2003).

[17] O. M. Boyarkin, G. G. Boyarkina and T. I. Bakanova, Phys. Rev. D 70, 113010 (2004).

[18] V. M. Abazov et al. [D0 Collaboration], Phys. Rev. Lett. 93, 141801 (2004).

[19] D. Acosta et al. [CDF Collaboration], Phys. Rev. Lett. 93, 221802 (2004).

[20] J. F. Gunion, J. Grifols, A. Mendez, B. Kayser and F. I. Olness, Phys. Rev. D 40, 1546 (1989).

[21] J. F. Gunion, C. Loomis and K. T. Pitts, arXiv:hep-ph/9610237.

[22] S. Chakrabarti, D. Choudhury, R. M. Godbole and B. Mukhopadhyaya, Phys. Lett. B 434, 347 (1998).

[23] J. F. Gunion, Int. J. Mod. Phys. A 13, 2277 (1998).

[24] B. Dion, T. Gregoire, D. London, L. Marleau and H. Nadeau, Phys. Rev. D 59, 075006 (1999).

[25] N. Arkani-Hamed, A. G. Cohen and H. Georgi, Phys. Lett. B 513, 232 (2001).

[26] J. F. Gunion, R. Vega and J. Wudka, Phys. Rev. D 42, 1673 (1990); R. Godbole, B. Mukhopadhyaya and M. Nowakowski, Phys. Lett. B 352, 388 (1995); K. M. Cheung, R. J. N. Phillips and A. Pilaftsis, Phys. Rev. D 51, 4731 (1995).

[27] A. Kundu and B. Mukhopadhyaya, Int. J. Mod. Phys. A 11, 5221 (1996).

[28] V. Barger, D. Marfatia and A. Tregre, Phys. Lett. B 595, 55 (2004).

[29] M. Czakon, M. Zralek and J. Gluza, Nucl. Phys. B 573, 57 (2000); M. Czakon, J. Gluza, F. Jegerlehner and M. Zralek, Eur. Phys. J. C 13, 275 (2000).

[30] M. C. Chen and S. Dawson, Phys. Rev. D 70, 015003 (2004); M. C. Chen, S. Dawson and T. Krupovnickas, arXiv:hep-ph/0504286. 
[31] T. Blank and W. Hollik, Nucl. Phys. B 514, 113 (1998); J. R. Forshaw, D. A. Ross and B. E. White, JHEP 0110, 007 (2001).

[32] E. Ma, M. Raidal and U. Sarkar, Phys. Rev. Lett. 85, 3769 (2000); E. Ma, M. Raidal and U. Sarkar, Nucl. Phys. B 615, 313 (2001).

[33] M. C. Chen, Phys. Rev. D 71, 113010 (2005).

[34] K. Hasegawa, Phys. Rev. D 70, 054002 (2004).

[35] A. Datta and A. Raychaudhuri, Phys. Rev. D 62, 055002 (2000).

[36] J. Abdallah et al. [DELPHI Collaboration], Eur. Phys. J. C 31, 421 (2004); G. Abbiendi et al. [OPAL Collaboration], Eur. Phys. J. C 32, 453 (2004).

[37] T. Han, H. E. Logan, B. Mukhopadhyaya and R. Srikanth, arXiv:hep-ph/0505260.

[38] G. Azuelos, K. Benslama and J. Ferland, arXiv:hep-ph/0503096.

[39] D. Acosta et al. [CDF Collaboration], arXiv:hep-ex/0503004.

[40] M. Muhlleitner and M. Spira, Phys. Rev. D 68, 117701 (2003).

[41] S. S. D. Willenbrock, Phys. Rev. D 35, 173 (1987); A. Krause, T. Plehn, M. Spira and P. M. Zerwas, Nucl. Phys. B 519, 85 (1998).

[42] J. Maalampi and N. Romanenko, Phys. Lett. B 532, 202 (2002).

[43] K. Huitu, J. Maalampi, A. Pietilä and M. Raidal, Nucl. Phys. B 487, 27 (1997).

[44] Q. H. Cao, S. Kanemura and C. P. Yuan, Phys. Rev. D 69, 075008 (2004); S. Kanemura and C. P. Yuan, Phys. Lett. B 530, 188 (2002).

[45] J. Pumplin, D. R. Stump, J. Huston, H. L. Lai, P. Nadolsky and W. K. Tung, JHEP 0207, 012 (2002); D. Stump, J. Huston, J. Pumplin, W. K. Tung, H. L. Lai, S. Kuhlmann and J. F. Owens, JHEP 0310, 046 (2003).

[46] A. D. Martin, R. G. Roberts, W. J. Stirling and R. S. Thorne, Eur. Phys. J. C 23, 73 (2002).

[47] Z. Maki, M. Nakagawa and S. Sakata, Prog. Theor. Phys. 28, 870 (1962).

[48] B. Aharmim et al. [SNO Collaboration], nucl-ex/0502021.

[49] Y. Ashie et al. [Super-Kamiokande Collaboration], Phys. Rev. Lett. 93101801 (2004).

[50] T. Araki et al. [KamLAND Collaboration], Phys. Rev. Lett. 94, 081801 (2005); M. Apollonio et al. [CHOOZ Collaboration], Eur. Phys. J. C 27, 331 (2003); F. Boehm, et al. [Palo Varde Collaboration], Phys. Rev. D 64, 112001 (2001). 
[51] M. A. Diaz, M. A. Garcia-Jareno, D. A. Restrepo and J. W. F. Valle, Nucl. Phys. B 527, 44 (1998); D. Aristizabal Sierra, M. Hirsch, J. W. F. Valle and A. Villanova del Moral, Phys. Rev. D 68, 033006 (2003). 